\title{
Information Aggregation and Strategic Abstention in Large Laboratory Elections
}

\author{
By Marco Battaglini, Rebecca B. Morton, and Thomas R. Palfrey*
}

Recent advances in voting theory have shed light on the influence of pivotality on voter choices when voters have asymmetric private information, and the implications of this for information aggregation in committees and elections. Of particular interest is the result that voters may optimally choose to vote contrary to their own private information, even in committees or elections where all voters share the same preferences (David Austen-Smith and Jeffrey Banks 1996). A related insight is that abstention can occur even when voting is costless, as in the so-called "Swing Voter's Curse" literature (Timothy Feddersen and Wolfgang Pesendorfer 1996). The reason is that private signals give voters information about the marginal distribution of states (given by voter signals), but what matters for an optimal decision is the distribution of states conditional on a pivotal event. For example, the pivotal event under majority rule arises when the aggregate votes of the other voters is either a tie or one vote away from a tie. These conditional distributions can be much different from the unconditional distribution of states. Because of these differences, some results are quite unintuitive and seem behaviorally implausible at first blush. Because these results have important implications about information aggregation and the efficiency of

\footnotetext{
* Battaglini: Department of Economics, Princeton University, Princeton, NJ 08544 (e-mail: mbattagl@princeton. edu); Morton: Department of Politics, NYU, New York, NY 10012 (e-mail: rebecca.morton@nyu.edu); Palfrey: Division of the Humanities and Social Sciences, California Institute of Technology, Mail Code 228-77, Pasadena, CA 91125 (e-mail: trp@hss.caltech.edu). We acknowledge financial support from the National Science Foundation (SES-0418150, SES-0617820, and SES-0450712), the Gordon and Betty Moore Foundation, PLESS at Princeton, CESS at NYU, and SSEL at Caltech. We also thank Stephen Coate, participants at the 2006 Wallis Political Economy Conference, the 2007 meetings of the Public Choice Society, David Austen-Smith and Massimo Morelli for comments. Rajeev Advani, Anna Bassi, Karen Kaiser, Kristin Michelitch, Uliana Popova, Anwar Ruff, and Stephanie Wang provided valuable research assistance.
}

election outcomes and committee decisions (Feddersen and Pesendorfer 1997, 1999), there is a need to test these theories, especially with respect to environments where the predictions seem implausible.

More generally, the view that voters condition their choices on pivotality remains controversial, especially among political scientists. ${ }^{1}$ Due to many confounding factors, attempts to test these theories by empirical study of voter behavior have been quite limited. For example, a number of researchers have used historical and survey data to establish a correlation between information and turnout (Palfrey and Keith Poole 1987; Thomas Coupe and Abdul Noury 2004; Matthew Gentzkow 2005, and others), but establishing a causal link has been more difficult (David Lassen 2005). Moreover, such a relationship might also be consistent with a simpler decision-theoretic model (John Matsusaka 1995). As Lassen specifically points out (p. 116), observational data are not rich enough in variation, nor do they provide researchers with enough controls to evaluate empirically the nuances of the pivotal voter approach. The laboratory may be the only place where such a study is possible.

This difficulty suggests a valuable role for laboratory experiments, where confounding factors can be eliminated and the environment can be controlled in order to obtain separation between the predictions of equilibrium theories based on game theory and pivotality versus nonequilibrium theories based on traditional decision theory. A few experimental papers have appeared in the literature in the last decade, testing pivotal voter models, ${ }^{2}$ but many open questions remain,

\footnotetext{
${ }^{1}$ See Donald Green and Ian Shapiro's (1996) critical point of view. Jeffrey Friedman (1996) contains a collection of articles addressing this controversy, and Feddersen (2004) reviews related literature pertaining specifically to abstention.

${ }^{2}$ See, for example, Serena Guarnaschelli, Richard McKelvey, and Palfrey (2000), Battaglini, Morton, and
} 
including questions about the generalizability of these findings to large elections.

This paper focuses squarely on the question of how these findings may change when the number of voters is scaled up. Because these models are intended to apply to both relatively small committees and mass elections (and everything in between), answering the scaling-up question is essential to understanding the general applicability of the theory.

We explore the scaling-up question with respect to one specific application of the theory, the Swing Voter's Curse (SVC). The SVC refers to a situation in which a voter, conditioning on being pivotal, may rationally choose to vote against his prior, or may abstain even if his prior clearly favors a given alternative. To see why this may happen, imagine a situation in which alternative $a$ is superior to alternative $b$, given prior information. Assume that most voters vote without observing which alternative is ex post superior (uninformed voters); some voters, however, have access to a private signal which reveals the true state (informed voters). In this case, it is not possible that the uninformed voters, following their prior, vote for $a$. If this were the case, an uninformed voter would indeed realize that, conditional on being pivotal, some votes must be cast by informed voters (or else $a$ would win for sure): this uninformed voter would not vote for $a$, because $b$ would certainly be a better alternative. As we will illustrate below, by a similar argument, uninformed voters may choose to vote against their prior if they know that there are partisan voters who would always favor $a$ regardless of the state. In these cases, therefore, the way voters make choices depends not only on their preferences and their information, but also on the entire distribution of preferences and information.

Battaglini, Morton, and Palfrey (2007b) present the first experimental study of the SVC and find significant evidence in support of the theory. They consider small committees, and find that uninformed swing voters abstain rather than cancel out more informed voters, even though voting costs are zero. Even more striking, in asymmetric environments, where there is partisan bias, these uninformed swing voters vote to offset these biases, even when their information says to vote in the same direction as the bias.

However, the experiments are conducted only on small committees of seven swing voters and generally six or fewer uninformed voters. In our scaled-up elections, we have between two and a half and three times as many voters of all kinds in each electorate. Other experimental studies have reported that voters in larger groups abstain with lower frequency than theory would suggest, even when the theory is modified to allow for some behavioral limitations. For example, David Levine and Palfrey (2006), in a study of costly voting, find that as they increase the size of the voting population, turnout rates are higher than the predictions of the Bayesian-Nash equilibrium, and also quantal response equilibrium (McKelvey and Palfrey 1995). It may be that as the size of the voting population increases, the predictions of the SVC find less support in the laboratory if swing voters participate more in such larger voting groups. We find, however, even in the larger voting groups, considerable support for the SVC and the pivotal voter theory of information aggregation in committees and elections.

\section{The Model and Equilibrium}

We consider a game with a set of $N$ voters who deliberate by majority rule. There are two alternative policies (or candidates) $a, b$, and two states of the world, $A$ and $B$. A number $m<N$ voters are partisans, who strictly prefer policy $A$ regardless of the state. For convenience, we assume that $m$ is even, $N$ is odd, and $m \leq N-3{ }^{3}$ The remaining $n=N-m$ voters are independents. These voters share common preferences represented by a utility function $u(x, \theta)$ that is a function of the state of the world $\theta \in\{A, B\}$ and the policy $x \in\{a, b\}$, where $u(a, A)=u(b, B)$ $=1$ and $u(a, B)=u(b, A)=0$. State $A$ has a prior probability $\pi \geq 1 / 2$. The true state of the world is unknown, but each voter may receive an informative signal. We assume that signals of different agents are conditionally independent. The signal can take three values $\alpha, \beta$, and $\varnothing$, with probabilities

$$
\begin{gathered}
\operatorname{Pr}(\alpha \mid A)=\operatorname{Pr}(\beta \mid B)=p \\
\text { and } \operatorname{Pr}(\varnothing \mid A)=\operatorname{Pr}(\varnothing \mid B)=1-p .
\end{gathered}
$$

\footnotetext{
${ }^{3}$ These assumptions are made to simplify the notation. 
An independent voter, therefore, is perfectly informed on the state of the world with probability $p$ (i.e., observes $a$ or $b$ ) and has no information with probability $1-p$ (i.e., observes $\varnothing$ ).

After voters have seen their private signal, all voters vote simultaneously. Each voter can vote for $a$, vote for $b$, or abstain $(\phi)$, and voting is costless. Partisans always vote for $a$. In any equilibrium in weakly undominated strategies, an independent who receives an $\alpha$ signal votes for $a$, and an independent who receives a $\beta$ signal votes for $b$. Therefore, in this model the only strategic voting comes from the uninformed independents. Let $\sigma_{a}, \sigma_{b}$, and $\sigma_{\phi}$ be the probability that an uninformed agent votes for $a$, for $b$, or abstains, respectively. An equilibrium of this game is symmetric if agents with the same signal use the same strategy: $\sigma^{i}=\sigma$ for all $i$. We analyze symmetric equilibria in which agents do not use weakly dominated strategies and we will refer to them simply as equilibria.

Battaglini, Morton, and Palfrey (2007b) characterizes the equilibria of the voting game, which is unique for the experimental parameters. Formal derivations and proofs appear in Battaglini, Morton, and Palfrey (2007b). First consider the benchmark case in which all the voters have the same common value, so $m=$ 0 . For this case, we have, $\sigma_{b}=0$ for all values of $\pi$. For values of $\pi$ sufficiently close to $1 / 2$ and $p$ sufficiently large, we also have $\sigma_{a}=0$, so all uninformed voters abstain. In the experiment, we choose parameters such that $\sigma_{a}=\sigma_{b}=0$ when $m=0$.

This equilibrium has a simple interpretation as a particular form of the SVC. To see the intuition behind it, suppose the prior is $\pi=1 / 2$. If an uninformed voter were to choose in isolation, he would be indifferent between the two options, $a$ or $b$. When voting in a group, however, he knows that with positive probability some other voter is informed. By voting, he risks voting against this more informed voter. So, since he has the same preferences as this informed voter and he is otherwise indifferent among the alternatives because he has no private information on the state, he always finds it optimal to abstain. When the prior is $\pi>1 / 2$, the problem of the voter is more complicated. In this case the swing voter's curse is mitigated by the fact that the prior favors one of the two alternatives. As before, the voter does not want to vote against an informed voter. He is not sure, however, that there is an informed voter: and if no informed voter is voting, he strictly prefers alternative $a$ since this is ex ante more likely. Thus, although the voter never finds it optimal to vote for $b$, he may find it optimal to vote for $a$. The higher is $\pi$, the higher is the incentive to vote for $a$; the higher is $p$ (i.e., the probability that there are other informed voters), the lower is the incentive to vote. For any $p$, if $\pi>1 / 2$ is not too high, the voter abstains.

\section{A. Partisan Bias}

If $m>0$, the analysis is more subtle. Now, uninformed voters always have an incentive to vote to balance out the partisans, who (from the standpoint of an independent) bias the outcome in favor of $a$. The calculus now depends critically on the conditional state probabilities if there is a tie. For example, if there is a tie, it means some of the independent voters have voted for $b$. Hence, in any equilibrium, $\sigma_{b}>0$ : i.e., uninformed voters must be voting for $b$ with positive probability. To see the logic, suppose not, so $\sigma_{b}=0$. This implies that if a pivotal event occurs, it must be that all the $b$ voters are informed independents, and hence the state is $B$. Therefore, the best response is $\sigma_{B}=1$, a contradiction. In addition, it is easy to show that there cannot be an equilibrium.

As in the case with $m=0$, if $\pi=1 / 2$ or if $\pi>1 / 2$ and $p$ is sufficiently large, then $\sigma_{a}=0$. The only equilibrium involves mixing between $b$ and abstention, so the equilibrium is characterized by a single number, $\sigma_{b} \in(0,1]$. In our experiment, we choose parameters such that $\sigma_{b}$ $\in(0,1)$, and $\sigma_{a}=0$.

There are several comparative static properties of the parameters of the model, $m, p, n, \pi$. For example, the higher is the bias in favor of $A$, the higher is $\sigma_{b}$.

\section{Experimental Design}

We use controlled laboratory experiments to evaluate the theoretical predictions. Once a specific parametrization for $n, m, \pi$, and $p$ is chosen, the model described and solved in the previous section can be directly tested in the lab without changes. In all of the sessions of the experiment, we used $p=0.25$. We had two sessions each of two different treatments for the probability distribution of the state of the 
world: $\pi=1 / 2$ and $\pi=5 / 9$. Within each session subjects participated in three different treatments for partisan bias: $m=0,6$, and 12 . The number of independents was 21 in three of the sessions and 17 in one session (with $\pi=1 / 2$ ).

The symmetric undominated Bayesian equilibrium is unique for all parameter values used in the experiment. For all elections, $\sigma_{a}=0$. For all $m=0$ elections, $\sigma_{\phi}=1$. For all elections with $m>0, \sigma_{b} \in(0,1)$. Specifically, the equilibrium predictions are: $\sigma_{b}(\pi=1 / 2 ; m=6 ; n$ $=21)=0.33 ; \sigma_{b}(\pi=1 / 2 ; m=12 ; n=21)=$ $0.69 ; \sigma_{b}(\pi=1 / 2 ; m=6 ; n=17)=0.42 ; \sigma_{b}(\pi$ $=1 / 2 ; m=12 ; n=17)=0.88 ; \sigma_{b}(\pi=5 / 9 ; m=$ $6 ; n=21)=0.32$; and $\sigma_{b}(\pi=5 / 9 ; m=12 ; n=$ $21)=0.69$. We contrast these with the decision theoretic predictions, based on naïve voting, as, for example, in Matsusaka (1995). According to the naïve model, voters abstain unless there are consumption benefits to voting, and these consumption benefits are independent of pivot probabilities. The consumption benefits are derived from voting for a choice that yields the highest utility given their prior beliefs about the state. In our experiment, the predictions of that model are: $\sigma_{\phi}=1$ for $\pi=1 / 2$; and $\sigma_{a}>\sigma_{b}=0$ for $\pi=$ $5 / 9$. Moreover, since voters are not strategic, $\sigma_{a}$ is independent from the number of partisans $m$.

The experiments were all conducted at the Center for Experimental Social Science at New York University and used registered students from New York University. ${ }^{4}$ Four sessions were conducted - three with 22 subjects and one with 18 subjects. ${ }^{5}$ No subject participated in more than one session. Each session had three subsessions, each lasting ten elections. All subsessions used the same $\pi$, but different values of $m=0,6$, and 12 . We varied the sequence of $m$ across sessions to control partially for any sequencing or learning effects. That is, for each value of $\pi$, we conducted one session using the order $(12,6,0)$ and one session using the order $(0,6,12)$. In the analysis that follows, we label the first variation Partisans First variation and the second Partisans Last variation. In the two Partisans Last sessions and the Partisan First

\footnotetext{
${ }^{4}$ The instructional and payment procedures are the same as described in Battaglini, Morton, and Palfrey (2007).

${ }^{5}$ We planned four 22 -subject sessions, but were 4 subjects short in one of the sessions. In each session, one subject was paid $\$ 20$ to serve as a monitor. Partisan voters were computerized.
}

session with $\pi=5 / 9, n=21$, but in the Partisan First session with $\pi=0.5, n=17$. We discuss the implications of this difference below.

\section{Experimental Results}

\section{A. Aggregate Voter Choices}

Informed Voters.-Of the 2,400 voting decisions we observed, in 618 cases (26 percent) subjects were informed, that is, revealed a red or yellow ball. Across all treatments and sessions, these informed voters chose 99 percent as predicted; 99.7 percent of the time if a voter revealed a red ball, he or she voted for jar 1 (state $A$ ) and 98 percent of the time if a voter revealed a yellow ball, he or she voted for jar 2 (state $B$ ). We interpret this as indicating that subjects had a least a basic comprehension of the task.

\section{Uninformed Voters: Effects of Treatments} on Voter Choices.-Table 1 summarizes the choices of uninformed voters as compared to the equilibrium predictions. In all treatments, we find that uninformed voters abstain in large percentages compared to informed voters, and these differences are significant. We also find strong evidence that the majority of uninformed voters alter their voting choices as predicted by the SVC theory and contrary to the decisiontheoretic theory. When $m=0$, uninformed voters abstain in high percentages. However, with partisan bias, uninformed voters reduce abstention and increase their probability of voting for $b$. The changes are all statistically significant. ${ }^{6}$

Session, Ordering, and Learning Effects.Figure 1 presents the average choices of uninformed voters over time by session. First observe that there are sharp changes in behavior immediately following a change in partisan bias, as shown in Table 1. Second, there appear to be some differences related to the order of variation in partisans when $\pi=1 / 2$ : the probability of voting for $b$ is lower in the Partisans First treatment than in the Partisans Last treatment for all values of $m$. However, this difference is significant

\footnotetext{
${ }^{6}$ The $t$-statistics are 12.64 and 4.19 , respectively, for the case when $\pi=1 / 2$ and 9.82 and 9.12 , respectively, for the case when $\pi=5 / 9$.
} 
Table 1-Vote Percentages by Uninformed Voters (Predicted percentages in parentheses)

\begin{tabular}{lrcrrl}
\hline \hline & $m$ & Observations & $a$ & $b$ & Abstain \\
\hline$\pi=1 / 2, n=17$ & 0 & 135 & $6(0)$ & $7(0)$ & $87(100)$ \\
& 6 & 120 & $4(0)$ & $55(42)$ & $41(58)$ \\
$\pi=1 / 2, n=21$ & 12 & 127 & $10(0)$ & $76(86)$ & $13(17)$ \\
& 0 & 151 & $9(0)$ & $9(0)$ & $83(100)$ \\
$\pi=5 / 9, n=21$ & 6 & 158 & $20(0)$ & $42(33)$ & $38(67)$ \\
& 12 & 163 & $21(0)$ & $48(69)$ & $31(31)$ \\
& 0 & 304 & $10(0)$ & $2(0)$ & $88(100)$ \\
& 6 & 311 & $11(0)$ & $35(32)$ & $54(68)$ \\
& 12 & 313 & $18(0)$ & $62(69)$ & $21(31)$ \\
\hline
\end{tabular}

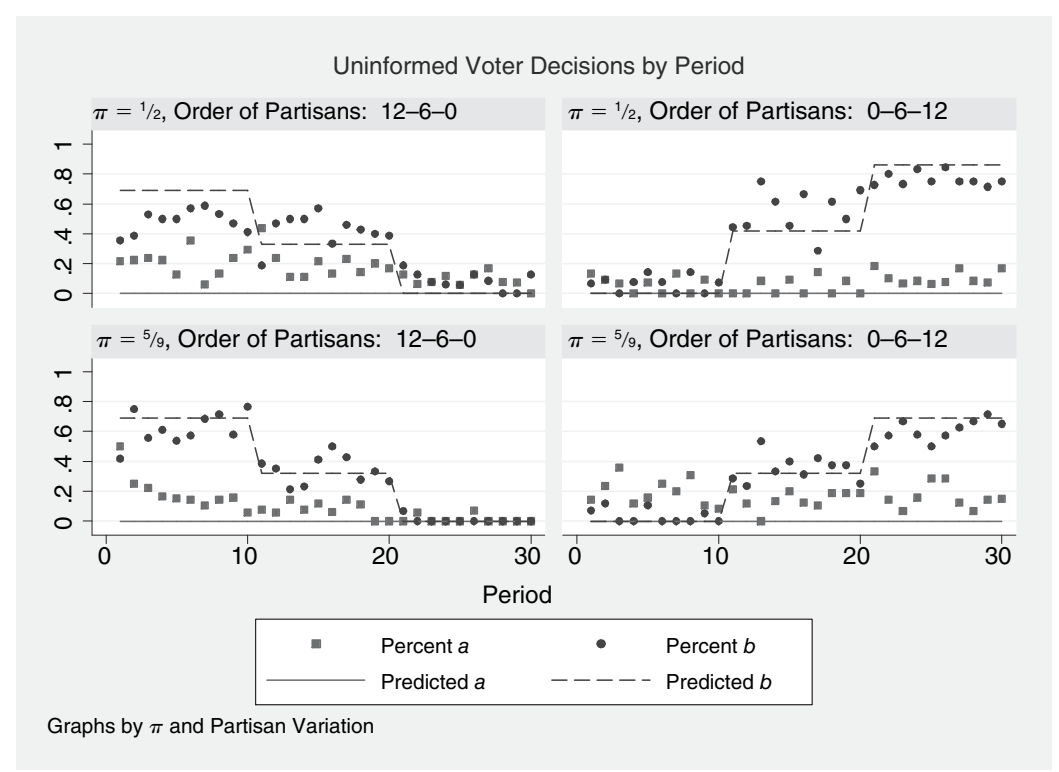

FIGURE 1

only when $m=6$ and $m=12 .^{7}$ This difference is expected since in the Partisans First treatment there were fewer voters, $n=17$, compared to $n=21$ in the Partisans Last session, so the predicted probability of voting for $b$ in the $n$ $=17$ session is greater, and this is reflected in the data. We also find differences between the two sessions when $\pi=5 / 9$. In particular, we see more voting in the Partisans Last variation than in the Partisans First variation. When $m$

\footnotetext{
${ }^{7}$ The $t$-statistics are $0.62,2.09$, and 5.12 for $m=0,6$, and 12 , respectively.
}

$=0$, uninformed voters are significantly more likely to vote for $b$ in the Partisans Last treatment than in the Partisans First treatment. ${ }^{8}$ Furthermore, when $m=0$ and 6, uninformed voters are significantly more likely to vote for $a$ in the Partisans Last variation. These differences appear to reflect differences in ordering of the treatments. ${ }^{9}$

\footnotetext{
${ }^{8}$ The $t$-statistic is 1.92 .

${ }^{9}$ The $t$-statistics are 5.33 and 1.85 for $m=0$ and 6 , respectively.
} 
Table 2-Mean Efficiency by Treatment

\begin{tabular}{lcrlc}
\hline \hline & State & Cases & Actual & Predicted \\
\hline$\pi=1 / 2, m=0$ & State $A$ & 11 & 1 & 1 \\
& State $B$ & 9 & 1 & 1 \\
$\pi=1 / 2, m=6$ & State $A$ & 14 & 0.46 & 0.98 \\
& State $B$ & 6 & 1 & 0.99 \\
$\pi=1 / 2, m=12$ & State $A$ & 15 & 0.27 & 0.93 \\
& State $B$ & 5 & 1 & 0.98 \\
$\pi=5 / 9, m=0$ & State $A$ & 14 & 1 & 1 \\
$\pi=5 / 9, m=6$ & State $B$ & 6 & 0.92 & 1 \\
& State $A$ & 7 & 0.93 & 0.99 \\
$\pi=5 / 9, m=12$ & State $B$ & 13 & 1 & 0.98 \\
& State $A$ & 9 & 0.33 & 0.84 \\
& State $B$ & 11 & 1 & 0.98 \\
\hline
\end{tabular}

\section{B. Efficiency of Choices}

Our analysis of voting behavior provides support for the SVC, but also shows that some uninformed voters vote for $a$. This deviation from optimal behavior should have the consequence of leading to less efficient choices for the group. We find that this is indeed the case when there are partisan voters, and for the treatment when $\pi=1 / 2$ where $n=17$ for half of the observations. However, when there are zero partisans, we find that 100 percent of the time when $\pi=1 / 2$, and 98 percent of the time when $\pi=5 / 9$, the group's choice is the correct one. When $m=6$ and $\pi=$ $1 / 2$, however, the group's choice is correct only 69 percent of the time. This increases to 93 percent of the time when $m=6$ and $\pi=5 / 9$, a difference which is significant at the 10 percent level. We find similar results for the comparison with $m=12$; when $\pi=1 / 2$, the group is correct only 39 percent of the time, while when $\pi=5 / 9$, the group's choice is correct 69 percent of the time, a difference that is significant at the 10 percent level as well.

Table 2 summarizes the mean efficiency results by the state of the world and treatment (with ties coded as 0.5 ) with the mean predicted efficiency given the number of informed voters in each period and predicted voting behavior.

\section{Concluding Remarks}

The central finding from this study is that most of the predictions of Swing Voter's Curse theory hold up in large elections conducted under controlled laboratory conditions. There is significant abstention, and significant balancing of partisans by uniformed voters; and vote balancing increases with the partisan imbalance. Elections with no partisan imbalance successfully aggregate information and lead to efficient outcomes. Consistent with SVC theory, this efficiency falls off as partisan imbalance increases, but to a significantly greater extent than is predicted in equilibrium.

It is instructive to compare these findings in large elections with results from the smaller elections reported in Battaglini, Morton, and Palfrey (2007b). All the qualitative results concerning the comparative statics, balancing, and abstention are the same. One slight difference is that there is less voting for $a$ in the small elections than in the large elections, except for the $\pi=5 / 9 m=0$ treatment, where we observed 20 percent voting for $a$ in the small elections, compared with 10 percent voting for $a$ in the large elections. These differences were reflected in slightly different efficiency results between small and large elections, with the comparisons mirroring the differences in voting for $a$ : more (irrational) $a$ voting results in lower efficiency.

We conclude that this scaled-up study successfully replicates the initial swing voter's curse experiment reported in Battaglini, Morton, and Palfrey (2007b), obtaining very similar findings in laboratory committees that are three times the size of those in the original study. The one caveat is that we found evidence of a slight increase in irrational nonequilibrium behavior (voting for $a$ ) in the larger elections. Whether this trend would continue as election size is further scaled up is an open question.

\section{REFERENCES}

Austen-Smith, David, and Jeffrey S. Banks. 1996. "Information Aggregation, Rationality, and the Condorcet Jury Theorem." American Political Science Review, 90(1): 34-45.

Battaglini, Marco, Rebecca B. Morton, and Thomas R. Palfrey. 2007a. "Efficiency, Equity, and Timing of Voting Mechanisms." American Political Science Review, 101(3): 409-24.

Battaglini, Marco, Rebecca B. Morton, and Thomas R. Palfrey. 2007b. "The Swing 
Voter's Curse in the Laboratory." California Institute of Technology Social Science Working Paper 1263.

Coupe, Tom, and Abdul G. Noury. 2004. "Choosing Not to Choose: On the Link between Information and Abstention." Economics Letters, 84(2): 261-65.

Feddersen, Timothy J. 2004. "Rational Choice Theory and the Paradox of Not Voting." Journal of Economic Perspectives, 18(1): 99-112.

Feddersen, Timothy J., and Wolfgang Pesendorfer. 1996. "The Swing Voter's Curse." American Economic Review, 86(3): 408-24.

Feddersen, Timothy, and Wolfgang Pesendorfer. 1997. "Voting Behavior and Information Aggregation in Elections with Private Information." Econometrica, 65(5): 1029-58.

Feddersen, Timothy J., and Wolfgang Pesendorfer. 1999. "Abstention in Elections with Asymmetric Information and Diverse Preferences." American Political Science Review, 93(2): 381-98.

Friedman, Jeffrey, ed. 1996. The Rational Choice Controversy: Economic Models of Politics Reconsidered. New Haven: Yale University Press.

Gentzkow, Matthew. 2006. "Television and Voter Turnout." Quarterly Journal of Economics, 121(3): 931-72.
Goeree, Jacob, and Leeat Yariv. 2007. "Conformity in the Lab." California Institute of Technology Working Paper.

Green, Donald, and Ian Shapiro. 1994. Pathologies of Rational Choice Theory. New Haven: Yale University Press.

Guarnaschelli, Serena, Richard D. McKelvey, and Thomas R. Palfrey. 2000. "An Experimental Study of Jury Decision Rules." American Political Science Review, 94(2): 407-23.

Lassen, David. 2005. "The Effect of Information on Voter Turnout: Evidence from a Natural Experiment." American Journal of Political Science, 49(1): 103-18.

Levine, David K., and Thomas R. Palfrey. 2007. "The Paradox of Voter Participation: A Laboratory Study." American Political Science Review, 101(1): 143-58.

Matsusaka, John G. 1995. "Explaining Voter Turnout Patterns: An Information Theory." Public Choice, 84(1-2): 91-117.

McKelvey, Richard D., and Thomas R. Palfrey. 1995. "Quantal Response Equilibria for Normal Form Games." Games and Economic Behavior, 10(1): 6-38.

Palfrey, Thomas R., and Keith T. Poole, 1987. "The Relationship between Information, Ideology, and Voting Behavior." American Journal of Political Science, 31(3): 511-30. 